\title{
Integrated Data Acquisition, Storage, Retrieval and Processing Using the COMPASS DataBase (CDB)
}

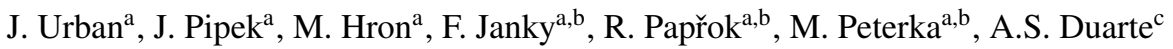 \\ ${ }^{a}$ Institute of Plasma Physics AS CR, v.vi., Za Slovankou 3, 18200 Praha 8, Czech Republic \\ ${ }^{b}$ Department of Surface and Plasma Science, Faculty of Mathematics and Physics, Charles University in Prague, V Holešovičkách 2, 18000 Praha 8, Czech \\ Republic \\ ${ }^{c}$ Instituto de Plasmas e Fusão Nuclear, Instituto Superior Técnico, Universidade Técnica de Lisboa, 1049-001 Lisboa, Portugal
}

\begin{abstract}
We present a complex data handling system for the COMPASS tokamak, operated by IPP ASCR Prague, Czech Republic [1]. The system, called CDB (Compass DataBase), integrates different data sources as an assortment of data acquisition hardware and software from different vendors is used. Based on widely available open source technologies wherever possible, CDB is vendor and platform independent and it can be easily scaled and distributed. The data is directly stored and retrieved using a standard NAS (Network Attached Storage), hence independent of the particular technology; the description of the data (the metadata) is recorded in a relational database. Database structure is general and enables the inclusion of multi-dimensional data signals in multiple revisions (no data is overwritten). This design is inherently distributed as the work is off-loaded to the clients. Both NAS and database can be implemented and optimized for fast local access as well as secure remote access. CDB is implemented in Python language; bindings for Java, $\mathrm{C} / \mathrm{C}++$, IDL and Matlab are provided. Independent data acquisitions systems as well as nodes managed by FireSignal [2] are all integrated using CDB. An automated data post-processing server is a part of CDB. Based on dependency rules, the server executes, in parallel if possible, prescribed post-processing tasks.
\end{abstract}

Keywords: tokamak, CODAC, database, data management, data acquisition PACS: 52.55.Fa, 07.05.Kf, 07.05.Hd

\section{Introduction}

With the increasing volume and complexity of diagnostics and synthetic data needed for tokamaks or other pulsed experimental devices, the demands on the data storage system are becoming very challenging. Present technologies are often difficult to scale, either due to the used technologies or due to the internal architecture. Since the COMPASS tokamak (re)started its operation at IPP Prague [1], more and more issues related to data storage emerged. This fact, together with the experience from other tokamaks, motivated the development of the COMPASS DataBase (CDB) - a system to be used to store and retrieve any (COMPASS) tokamak related numerical data.

This paper first discusses the fundamental goals and motives of CDB in Section 2 The CDB architecture is described in Section 3. The core functionality is implemented in Python with bindings for many different languages and interfaces, as described in Section 4 . The integration of the various data acquisition systems is addressed in Section 5 A recently implemented automatic post-processing capability is described in Section 6 Finally, Section 8 gives our conclusions.

\section{Goals and motivation}

As already noted above, it was the re-installation of the COMPASS tokamak at IPP Prague that provided the fundamental motivation for the development of a new data storage system.
Within a close collaboration with IPFN Lisbon, a new CODAC (Control, Data Access and Communication) system [3, 4] has been built. In the beginning, the system consisted of ATCA data acquisition hardware and FireSignal [2], which controlled the data acquisition nodes. SDAS [5] was used to access the data provided by FireSignal. This original design was strongly focused on data acquisition as the data signals were identified by hardware identifiers while the natural identification is the (measured) physical quantity. Moreover, data are written to and read from a central server, which can thus become overloaded. This is the case of COMPASS as around 2 GB of diagnostic data are presently produced for a single discharge; this number can become several times larger in the near future. Moreover, the API was not very flexible and numerical data were stored in custom binary files.

COMPASS DataBase (CDB) has been designed to enhance the capabilities and performance of COMPASS CODAC, focusing mainly on the final storage system. CDB provides a way to write data from data acquisition nodes in parallel, without a central collection point. As such, the FireSignal central server can be used for nodes control only, while the data are written directly to CDB. CDB is easily scalable, portable and extensible. Although targeted to be used primarily on COMPASS, the aim is to create a universal tool. The CDB architecture is depicted in Fig. 1 and described in detail in the following. 


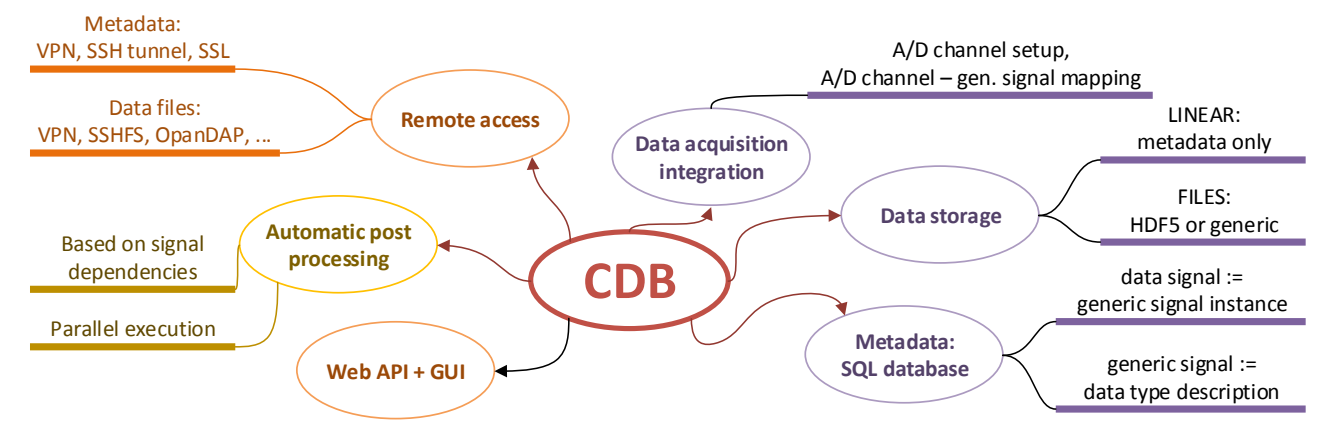

Figure 1: An overview diagram of CDB components.

\section{The architecture of CDB}

\subsection{Data and metadata}

Scientific data that we need to store are well structured (we know in advance the type, the dimensionality, etc.) and can be decomposed into actual data (numbers) and metadata, which contain the information about the data content (e.g., the physical quantity, units, etc.).

The data model of CDB, depicted in Fig. 2, is based on generic signals, which contain the descriptions of possible data types that can be stored, and data signals, which are instances (realizations) of generic signals and contain the metadata of a particular dataset, including the data file, in which the numerical data are stored. Generic signal description also contains the axes, which are generic signals as well.

A single data signal as well as single data file always belong to a particular record number and can exist in one or more revisions. The record number denotes either a particular experimental discharge or a model (simulation) or a void (e.g. a trigger test) record.

Two types of data signals can be stored in CDB: FILE and LINEAR. FILE signals have the data in data files while LINEAR signals are described by a linear function. Linear transforms of FILE signals can also be stored, particularly for converting from data acquisition levels to physical units or for correcting the data, so that no new data file has to be created.

All metadata, i.e., generic and data signals, records, data file descriptions and more, are stored in a relational database, particularly MySQL, although a different database engine can be used. The numerical data are stored in files on a network attached storage (NAS). The primary file format is HDF5 [6]. There are many good reasons to employ HDF5 files on a NAS as the numerical data storage back end. HDF5 is one of the most enhanced and wide spread file formats, which allows to conveniently read and write almost any kind of numerical datasets, organized hierarchically in a file. Very importantly, HDF5 API is extremely rich in its functionality and is available for all relevant programming languages. Each HDF5 file can contain one or more CDB data signals. CDB also allows to store any other data type, however, without providing any compatibility to read the data. This feature can be convenient, for example, for storing whole custom data files of a simulation code (instead of storing it "somewhere", CDB stores the file and its metadata in a well defined place) or for storing encoded videos or images.

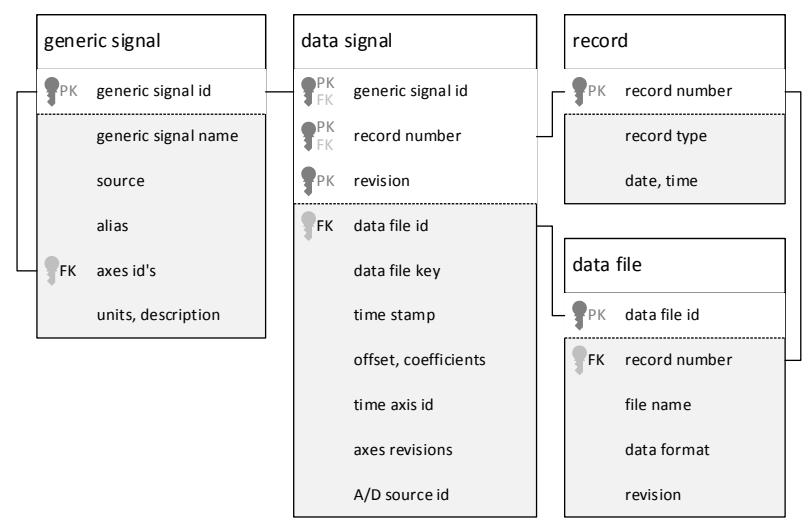

Figure 2: A simplified diagram of the CDB data model. We use an SQL-like graphics since the data structure is directly reflected in the SQL schema. Keys denote primary identifiers (primary keys in SQL), lines denote references (SQL foreign keys). Certain fields, e.g. axes id's, represent multiple fields in reality.

Since CDB uses conventional file access protocol, any NAS can be used to read and write the data files. This is, in most cases, a big advantage as the storage can be tailored and optimized independently of CDB itself. A cluster storage system is actually used for COMPASS [7]. To increase the performance of writing data from FireSignal, we have an additional local cache on the central server.

\subsection{Signal identifiers}

CDB generic signals are uniquely identified either by their numeric id, by a combination of name and data source or by an alias. In order to have a unified API for different languages and for different (and extensible) identification schemas, CDB uses string identifiers. We define gs_str_id (generic signal string id) as a string that contains one of the unique generic signal identifiers.

Data signals are uniquely identified by a unique generic signal identifier (see above) in combination with a record number and a revision. For this reason, we define a string identifier str_id:

str_id :=

<CDB:> gs_str_id <:record_number <:revision>> < [units]> |

FS | DAQ: channel_id <:record_number <:revision>> < [units] > where

channel_id := computer_id/board_id/channel_id 


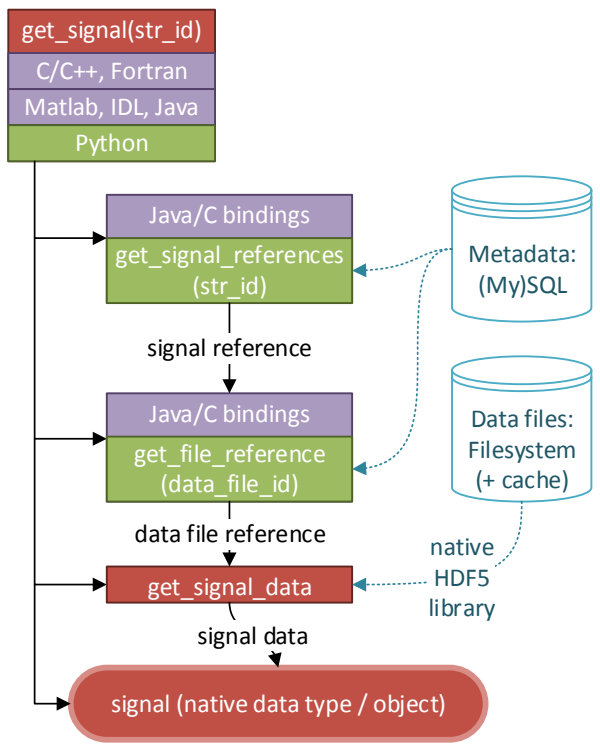

Figure 3: A schema of getting a signal from CDB. The starting point is the get_signal function in various languages. Core Python functions (green) are called via different APIs. Dotted lines show the use of back-end technologies. Finally, the data are assembled into a signal data type, which is native for the calling language.

Here CDB, FS and DAQ are schemas for signal identification. The default is the native CDB schema while FS and DAQ are used for identification by data acquisition channels using FireSignal or CDB native id's, respectively. The following str_id examples refer to the same signal, the first one by its alias while the others by its DAQ and FireSignal id's:

1. I_plasma: 4073:-1 [default]

2. DAQ:ATCA_1/9/13:-1

3. FS:PCIE_ATCA_ADC_01/BOARD_9/CHANNEL_013:4073

String identifiers can be used in any programming language, hence simplifying and unifying the bindings, and are easy to compose and parse. They can also be straightforwardly extended for, e.g., multiple tokamaks (by adding the tokamak name as a prefix), different databases or even indexing and slicing. An additional schema can be implemented if needed. In particular, an ITM CPO [8] schema is planned to be implemented, which would allow to search signals by CPO field names.

\subsection{Data safety and consistency, revisions}

When a new version of a signal is stored, e.g., in case of an error, a new revision is stored instead of overwriting the existing data. As such, full history is saved and data consistency and persistence is assured. When a data file is closed, CDB client changes its permissions to read only. Moreover, the cache mechanism changes the owner to root and moves the files to the permanent storage, which can be read-only for clients.

\subsection{Data reading and storing}

CDB provides a simple data access along with a comprehensive set of functions for specific use cases. This is particularly possible without too much effort due to the use of
HDF5 files. For simple data reading, function get_signal (str_id) reads a data signal including all axes and numerical data, as shown in Fig. 3. This function returns either an object or a structure depending on the programming language. Alternatively, we can request only signal reference(s) (i.e. the metadata) and use the versatile HDF5 API to access the data. In our opinion, using HDF5 is extremely beneficial as we save the tremendous effort that would be needed to reimplement similar functionality. Besides simple dataset reading and writing, HDF5 natively supports reading portions of datasets (hyperslabs), compound data types, point selection, extensible datasets, parallel access and even more.

Storing data is naturally more complex. In general, the following steps are required to store data into CDB. Function store_signal is used in steps 6-7. Its parameters are basically the fields from the data signal description (see Fig. 2).

1. Get the record number or create a new record.

2. Get the generic signal reference for the data to be stored.

3. Create a new data file-file path and name are obtained.

4. Write the numerical data into this file. Axes data can be written into the same file if convenient.

5. Close the file because CDB needs to know when writing to the file has finished.

6. Either store the axes data signals or get their references if axes signals are already available. This must precede the next step because we must know the axes data signal revisions.

7. Store the main data signal.

Steps 3-5are needed for FILE signals only.

Frequently, a simplified put_signal function can be used, particularly when storing new numerical data together with the axes. Another convenience function is update_signal, which creates a new revision of a signal by changing only the metadata (offset, coefficients, time axis, axes id's). Technically, this function replaces step 3 by getting the reference of the signal to be updated, skips steps 4-6 and finally stores the signal using the new metadata.

\subsection{Remote access}

Remote access to CDB is provided by making accessible the data and metadata storage and configuring the CDB client accordingly by specifying the SQL server address and the data files root directory. There are no specific parts of the CDB code base dedicated to remote access. Available solutions, such as $\mathrm{SSH}, \mathrm{VPN}$ or global file systems, can readily be used.

\subsection{Data acquisition set up}

CDB is capable to keep track of the relations between data acquisition channels and generic signals along with a text description of the configuration (e.g. the configuration file). In other words, CDB knows what quantity is acquired by a particular A/D channel. This information can be very important since almost thousand channels are installed. Consequently, stored signals can be referenced either by generic signals or by $A / D$ channel id's. 


\section{Implementation details}

\subsection{Core functionality}

The core functionality is implemented in Python. Main reasons why Python is used are:

(a) Python is an open source, universal, widely used programming language.

(b) Python is well equipped for interfacing to other languages.

(c) Development in Python is rapid, which is supported by a huge set of available libraries.

(d) The native API can be readily used for data analysis and graphics, for which Python is well equipped.

In fact, from the experience with $\mathrm{CDB}$ and other projects, we can state that Python is presently the most capable and versatile open source, high-level programming for scientific use.

\subsection{Bindings to other languages}

CDB bindings to other languages are conveniently provided using Jython and Cython. Jython is a Python implementation in Java; however, it can be equally well used to execute Python from Java. Similarly, the primary purpose of Cython is writing $\mathrm{C}$ extensions in a Python-like language but it is also possible to create C API to Python functions.

Matlab and IDL are frequently used (commercial) products for scientific programming and data analysis. They can both call $\mathrm{C}$ functions by creating special wrappers; however, this capability is not very convenient, particularly in case of IDL, in which it is extremely user unfriendly. There are also problems with dynamically linked libraries. Finally, after implementing basic CDB bindings for Matlab and IDL using $\mathrm{C}$ and Cython, we rather decided to develop a Java-based interface. While Matlab runs Java natively, IDL is sometimes more peculiar. Nevertheless, the Python-Jython-Java solution proved to be most convenient and well maintainable for both Matlab and IDL, as well as for any other Java-based product.

An important feature of the CDB design is that the terminal languages use the bindings for metadata reading and writing only. Numerical data, i.e., HDF5 files in most cases, are read and written with native functions directly from/to the final file storage (NAS). This enhances the performance and relaxes the demands on servers. Although this solution implies that some parts of the code must be duplicated, the performance gain is more important.

If a programming language is required that cannot incorporate Python, Java or $\mathrm{C} / \mathrm{C}++, \mathrm{CDB}$ provides web services that map to CDB API and use JSON as the data format. Although the full potential of this interface has not been fully exploited yet, it clearly enables, e.g., the creation of various web tools.

\section{Data acquisition integration with CDB}

On COMPASS, around 1000 data acquisition channels of different hardware characteristics as well as various modes of operation are installed. Among them are general-purpose multichannel systems with public hardware drivers and C-API (ATCA boards), machines supporting remote network protocols (d-tAcq 216 or 196), hardware requiring specific platform-dependent software controllable through a custom IPC protocol (infrared camera) or hardware controlled using applications written in very specific programming languages. Some of them produce 1-dimensional data with linear time axes, others produce multidimensional data with irregular samplings in time and space. Although there is no single way to manage all these data-producing agents, most of them have been easily integrated into CDB because of its flexibility and language independence.

\subsection{FireSignal}

Most data acquisition systems are controlled through FireSignal [2], a client-server network system. In its original form, this system provides a central server which is the hub of all communication-all other components connect to it through CORBA and do not communicate with each other. A database controller stores and retrieves data, which are provided by nodes. Users can connect via a graphical interface.

This architecture follows best design principles in that it conceptually divides the responsibility to subsystems and clearly separates the problem of data storage from the problem of control and the data acquisition. However, in the case of COMPASS, this elegance of design brings about a few disadvantages, most severe being the heavy load of the central server (most of which is just passing data around). Therefore (and because of the change of the underlying database solution), we adopted a few changes that violate the fundamental FireSignal architecture but which lead to higher efficiency and employ CDB's potential:

(a) The database controller stores data into CDB (using Jython). Time axes, scaling coefficients and generic signals are automatically assigned in this step.

(b) Data in the client GUI are read directly from CDB instead of being requested and received through central server.

(c) There is a mechanism for the nodes to write data directly to CDB (using either Python, Cython or Jython, depending on the node implementation language) instead of sending them to the central server (and database controller). In such case, the nodes just inform the central server that they finished writing.

(d) Multidimensional data and data without any time axis can be written from the nodes.

The final goal is to write as much data as possible directly from the nodes to CDB. For this purpose, we have developed $\mathrm{C}++$ and Java libraries. Nevertheless, the implementation of each node brings about specific complications. In any case, the fall-back indirect mechanism of data storing through central server will remain possible.

\subsection{Independent data acquisition sources}

Certain data acquisition systems do not easily fit in the FireSignal work flow, for which a special node is either very difficult (because of the API language or the operating system) or 
impractical (because of the way they are configured or used between discharges). However, if these systems know when to collect data, how to collect data and where to write data, the data acquisition can be handled easily using triggers and the CDB interface.

\section{Automatic data post-processing}

Numerous data post-processing tasks need to be executed after each tokamak discharge, ranging from a simple noise or drift removal to a magnetic equilibrium reconstruction. Moreover, it is extremely important to have the post-processed data consistent with the source data. If, for example, a source signal is updated (a new revision is created), the processing must be re-executed. As there seems to be no well suited solution available, we have decided to incorporate a post-processing capability into CDB.

Each CDB post-processing task has specified input and output signals. Tasks and their input and output signals form a directional graph, in which each task has edges oriented from the input signal nodes and to the output signal nodes. The graph must be acyclic, i.e., iteration over multiple tasks is not allowed. In addition, each output signal must be created by exactly one task, otherwise dependencies are ambiguous. Postprocessing tasks are stored in an SQL table and the prerequisites are checked whenever a new task is added.

Tasks are implemented as Python scripts, which either contain all the functionality or call external programs. The tasks are executed by traversing the tasks graph. IPython.parallel is employed to execute the tasks in parallel. Running processes are periodically checked and new tasks are executed whenever all necessary inputs become available. In addition, log entries are created, which allows to analyse the execution and also to execute the tasks only if newer inputs are available.

The post-processing is currently in a testing phase and launched manually. In the future, it will be triggered by storing a signal so that the post-processed signals will always be up to date.

\section{A brief comparison to existing systems}

Two most similar products used in the tokamak community are IDAM [9] and MDSPlus [10]. A notable difference is that CDB does not enforce a specific data access protocol (although HDF5 is de facto native) while IDAM and MDSPlus feature a unified local and remote access. CDB also directly exposes the meta data SQL server to clients, hence enabling custom queries. Composite signals (expressions) in IDAM (MDSPlus) can be replaced by the post-processing in CDB.

\section{Summary and conclusions}

A new data storage solution, the COMPASS DataBase (CDB), has been implemented for the COMPASS tokamak data. CDB integrates available and open technologies, such as HDF5 and MySQL, and provides a low-overhead, easily scalable and extensible solution for storing tokamak (or, generally, any pulsed device) experimental and simulation data. It has met the challenging needs of the COMPASS tokamak, particularly storing many data signals, which can originate from many different data acquisition systems, in a convenient, transparent, consistent and persistent form. CDB integrates easily with FireSignal as well as other systems, independent of their operating system or the API language. Data post-processing, described by signal dependencies, is conveniently automated. CDB is available under MIT license at http://bitbucket .org/compass-tokamak/ $\mathrm{cdb}$.

\section{Acknowledgement}

This work has been carried out within the framework of the Contract of Association between EURATOM and the IPP.CR. The views and opinions do not necessarily reflect those of the European Commission. The work of J. Urban was partially supported by Czech Science Foundation grant 13-38121P, the work of M. Hron and F. Janky by Czech Science Foundation grant P205/11/2470, the work at IPP AS CR by MSMT LM2011021.

\section{References}

[1] R. Pánek, O. Bilyková, V. Fuchs, M. Hron, P. Chráska, P. Pavlo, J. Stöckel, J. Urban, V. Weinzettl, J. Zajac, F. Žáček, Reinstallation of the COMPASS-D tokamak in IPP ASCR, Czechoslovak Journal of Physics 56 (2006) B125-B137.

[2] A. Neto, H. Fernandes, A. Duarte, B. B. Carvalho, J. Sousa, D. F. Varcarcel, M. Hron, C. A. F. Varandas, FireSignal - data acquisition and control, Fusion Engineering and Design 82 (2007) 1359-1364.

[3] D. F. Valcarcel, A. Neto, J. Sousa, B. B. Carvalho, H. Fernandes, J. C. Fortunato, A. S. Gouveia, A. J. N. Batista, A. G. Fernandes, M. Correia, T. Pereira, I. S. Carvalho, A. S. Duarte, C. A. F. Varandas, M. Hron, F. Janky, J. Pisacka, An ATCA embedded data acquisition and control system for the COMPASS tokamak, Fusion Engineering and Design 84 (2009) 1901-1904.

[4] M. Hron, F. Janky, J. Pipek, J. Sousa, B. B. Carvalho, H. Fernandes, P. Vondracek, P. Cahyna, J. Urban, R. Paprok, O. Mikulín, M. Aftanas, R. Panek, J. Havlicek, J. Fortunato, A. J. N. Batista, B. A. Santos, A. Duarte, T. Pereira, D. Valcarcel, Overview of the compass codac system, Fusion Engineering and Design (2013) doi:10.1016/j.fusengdes.2013.09.010.

[5] A. Neto, H. Femandes, D. Alves, D. F. Valcarcel, B. B. Carvalho, J. Ferreira, J. Vega, E. Sanchez, A. Pena, M. Hron, C. A. F. Varandas, A standard data access layer for fusion devices R\&D programs, Fusion Engineering and Design 82 (2007) 1315-1320.

[6] The HDF Group, Hierarchical data format version 5, 2000-2010.

[7] J. Písačka, M. Hron, F. Janky, R. Pánek, Cluster storage for COMPASS tokamak, Fusion Engineering and Design 87 (2012) 2238-2241.

[8] F. Imbeaux, J. B. Lister, G. T. A. Huysmans, W. Zwingmann, M. Airaj, L. Appel, V. Basiuk, D. Coster, L. G. Eriksson, B. Guillerminet, D. Kalupin, C. Konz, G. Manduchi, M. Ottaviani, G. Pereverzev, Y. Peysson, O. Sauter, J. Signoret, P. Strand, A generic data structure for integrated modelling of tokamak physics and subsystems, Computer Physics Communications 181 (2010) 987-998.

[9] D. G. Muir, L. Appel, N. J. Conway, A. Kirk, R. Martin, H. Meyer, J. Storrs, D. Taylor, N. Thomas-Davies, J. Waterhouse, MAST's integrated data access management system: IDAM, Fusion Engineering and Design 83 (2008) 406-409.

[10] G. Manduchi, Commonalities and differences between MDSplus and HDF5 data systems, Fusion Engineering and Design 85 (2010) 583-590. 